\title{
Effect of Different White-spot Lesion Treatment on Orthodontic Shear Strength and Enamel Morphology: In Vitro Study
}

\author{
Ari Triwardhani, Melisa Budipramana, Jusuf Sjamsudin \\ Department of Orthodontics, Faculty of Dental Medicine, Universitas Airlangga, Surabaya, Indonesia
}

\section{Abstract}

\begin{abstract}
Aim: White-spot lesion treatment is required before fixed appliance insertion as caries prevalence increases during orthodontic treatment. The aim of this study was to analyze the effect of fluoride varnish, casein phosphopeptide amorphous calcium phosphate fluoride (CPP-ACPF) varnish, and resin infiltration as white-spot lesion treatments on orthodontic shear strength and enamel morphology. Materials and Methods: This is an observational analysis study with posttest-only control group design and blind total sampling methods. Sixty bovine mandible incisors were cleaned and divided into five groups $(n=12)$. No treatment was applied on Group 1 (control), whereas the four other groups were immersed in demineralization solution for $96 \mathrm{~h}$ before being stored in artificial saliva (Group 2), treated with fluoride varnish (Group 3), CPP-ACPF varnish (Group 4), and resin infiltration (Group 5). After bracket bonding with resin-modified glass ionomer on all samples, shear bond strength was evaluated. Adhesive remnant index (ARI) was observed by using a digital photo. Enamel morphology was evaluated using a scanning electron microscopy (SEM) before bracket bonding and after polishing. The Kruskal-Wallis test $(P>0.05)$ followed by Mann-Whitney $U$ test was carried out $(P<0.05)$. Results: The shear strength in Groups 2 and 5 was slightly increased as compared with control group $(P>0.05)$, whereas it was significantly decreased in Groups 3 and $4(P<0.05)$. The ARI score was found to be highest after treatment with resin infiltration. Conclusion: Pretreatment with resin infiltration is recommended to be performed before bracket bonding on white-spot lesion; however, it may need to be reapplied after debonding and polishing to conceal enamel morphology damage formed after polishing.
\end{abstract}

Keywords: Enamel Morphology, Orthodontic Shear Strength, Orthodontic Treatment, White-spot Lesion Treatment

\section{INTRODUCTION}

One of the side effects of fixed orthodontic treatment is the formation of white-spot lesions (WSLs), defined as decalcification or demineralization of the enamel appearing as a chalky-white area on a tooth surface, which were considered as the early stage of dental caries caused by bacterial plaque activity. The WSLs at the end fixed orthodontic treatment prevalence has been mentioned in up to 97 of patients. ${ }^{[1]}$ A meta-analysis study revealed that the incidence of WSLs in patients with orthodontic appliances was $45.8 \%$, whereas its prevalence was found to be $68.4 \%{ }^{[2]}$ The presence of WSLs is strongly influenced by the level of oral hygiene, sex, orthodontic treatment length, flour intake, and diet..$^{[3]}$

In WSLs, a decrease in orthodontic bracket bonding strength occurs, which will cause the bracket to fall off

\begin{tabular}{|l|l|}
\hline \multicolumn{2}{|c|}{ Access this article online } \\
\hline Quick Response Code: & Website: \\
\hline & www.jioh.org \\
\cline { 2 - 2 } & \\
\hline
\end{tabular}

more easily during the course of treatment. It may be caused by the poor quality of enamel surfaces and lack of resin tag that makes it possible for mechanical attachment between composite bonding material and enamel surface to occur. This is very detrimental to orthodontists and patients as it prolongs the overall treatment time and the duration of dental visit. ${ }^{[4,5]}$

To overcome this problem, several agents that can inhibit enamel demineralization and enhance enamel

Address for correspondence: Dr. Ari Triwardhani Department of Orthodontics, Faculty of Dental Medicine, Universitas Airlangga, Campus A, Jl. Prof. Dr. Moestopo No. 47, Surabaya 60132, East Java, Indonesia. E-mail: vari-t@fkg.unair.ac.id

This is an open access journal, and articles are distributed under the terms of the Creative Commons Attribution-NonCommercial-ShareAlike 4.0 License, which allows others to remix, tweak, and build upon the work non-commercially, as long as appropriate credit is given and the new creations are licensed under the identical terms.

For reprints contact: reprints@medknow.com

How to cite this article: Triwardhani A, Budipramana M, Sjamsudin J. Effect of different white-spot lesion treatment on orthodontic shear strength and enamel morphology: In vitro study. J Int Oral Health 2020;12:120-8. 
remineralization can be used, especially the ones that can be applied in one visit to shorten the duration of the dental visit and do not require patient's cooperation. Fluoride, which is commonly used as a remineralization agent, forms a layer of calcium fluoride on the enamel surface, thus stimulating enamel remineralization. ${ }^{[6]}$ The application of fluoride varnish can significantly decrease the formation of the demineralization lesion around the bracket on anterior teeth. ${ }^{[7]}$ The addition of fluoride to casein phosphopeptide amorphous calcium phosphate fluoride (CPP-ACP) strengthens the remineralization effect as compared with the use of either fluoride or CPP-ACP. CPP-ACPF is able to provide calcium, phosphate, and fluoride ion reservoir on tooth surfaces to form a more acid-resistant fluorapatite film. ${ }^{[8]}$ In addition, WSLs can also be treated by the application of resin infiltration. Resin infiltration can significantly arrest the progression of early caries lesions by eroding and destroying the lesions, then coating them with triethyleneglycol-dimethacrylate-based resins, which increase the resistance of lesions and surrounding healthy enamel against acid. ${ }^{[9]}$ However, those treatments may affect the shear strength of the orthodontic bracket bonding.

On the basis of these facts, this research is conducted to determine the effect of several one-visit treatments of WSLs before orthodontic fixed treatment on shear strength of resin-modified glass ionomer (RMGIC) orthodontic bonding agent and their effect on enamel morphology.

\section{Materials and Methods \\ Study design}

This in vitro study was, using posttest-only control group design, carried out in Faculty of Dental Medicine Universitas Airlangga, Indonesia, in May-June 2017. Sixty samples of bovine (Bos indicus) extracted permanent mandibular incisors that meet inclusion criteria such as root formation completed, no caries/fracture/abrasion/ enamel damage, no enamel alteration, no immersion in chemical substances.

Studied teeth were cleaned using pumice on a low-speed handpiece brush to remove debris, then cut by separating disk (7/8 SS Flat Baker) mounted on a straight low-speed handpiece. The teeth were cut on the cervical section to separate the crown and the root. The cut tooth was then placed inside a preformed plastic tube with its labial surface facing upwards and at an equal height to the top of the plastic tube, using self-cured acrylic (Vertex) as its stabilizer. The plastic tube was made from a 5-cc syringe tube, with a screw half-inserted into it as it acted as a handle during shear strength test procedure.

\section{Study procedure}

The samples were then divided into five groups $(n=12)$. No treatment was applied on to Group 1 (control). Each sample in the other four groups was immersed in $10 \mathrm{~mL}$ of demineralization solution $(\mathrm{HCl} 33 \%, \mathrm{NaOH} 40 \%$, and dan $\mathrm{H}_{2} \mathrm{SO}_{4} 27 \%$ ) for $96 \mathrm{~h}$ in the incubator at $37^{\circ} \mathrm{C} \cdot{ }^{[10]}$ This immersion was performed to produce artificial caries lesion as deep as 120-200 $\mu \mathrm{m}$. Samples on Group 2 were then stored in artificial saliva. WSL treatments were performed on the other three groups: fluoride varnish (Fluor Protector, Ivoclar Vivadent) in Group 3, CPP-ACPF varnish (MI Varnish, GC) in Group 4, and resin infiltration (ICON Infiltrant, DMG) in Group 5. All treatments were performed according to the manufacturer's instruction. After treatments, all samples were put in artificial saliva until bracket placement.

RMGIC bonding agent was applied on maxillary central incisor bracket mesh (Integra, RMO) using an applicator stick. Brackets were then attached onto the teeth. The excessive adhesive agent was removed using a probe. The brackets were then cured using a light-curing unit from four directions, each for $10 \mathrm{~s}$. All samples were stored in artificial saliva for 1 day before shear strength test to make complete polymerization of RMGIC bonding agent possible.

\section{Observational Parameters}

Each sample was then placed on shear strength measurement tool (Autograph Shimatzu, Kyoto, Japan). After that, obtained data were analyzed using Kolmogorov-Smirnov one-sample normality test $(P>$ 0.05 ), one-way analysis of variance (ANOVA) test, and Tukey honestly significant difference (HSD) statistical test $(P<0.05)$. A visual scoring of adhesive remnant index (ARI) using digital camera was performed by three different observers using 4-point scale of Artun and Bergland $^{[11]}$, where 0 - no adhesive left on tooth surface; 1 -less than $50 \%$ of the adhesive is left on tooth surface;

2: more than $50 \%$ of the adhesive is left on tooth surface; and 3 -all adhesive is left on tooth surface with a distinct impression of the bracket base. ARI evaluation scores obtained were analyzed by Kruskal-Wallis test and Mann-Whitney $U$ test $(P<0.05)$.

Further visual observation on the enamel surface by scanning electron microscopy (SEM) was made before the application of white-spot treatments and after debonding and polishing. Crack length and porosity diameter were analyzed using the Kolmogorov-Smirnov one-sample normality test, one-way ANOVA test, and Tukey HSD statistical test. $t$-Test was used to analyze the difference of crack length before and after debonding and polishing.

\section{Statistical Analysis}

All statistical analyses were performed using the Statistical Package for Social Sciences software version 20.0 (IBM, Chicago, IL). Descriptive statistics were performed for the calculation of mean, standard deviation, maximum, and minimum. The Kruskal-Wallis test $(P>0.05)$ followed by Mann-Whitney $U$ test was carried out $(P<0.05)$. 


\section{RESULTS}

\section{Effect of treatments on enamel surface}

On $\times 50$ magnification of SEM observation [Figure 1], it was observed that enamel surface in the control group showed slight depressions with a defined margin. In the demineralization group, the enamel surface looked chalky with more clearly seen vertical depressions, some with defined margin. After fluoride varnish was applied, enamel depressions appeared to be filled with the material so that they were not as deep as those in demineralization group. In contrast, there were depressions found in CPP-ACPF varnish treatment group because it appeared to be concealed by a homogeneous layer. Treatment with resin infiltration caused the enamel surface to look smoother than that of control group.

It appeared that most of the directions of enamel cracks in the all groups were vertical and diagonal, but there were also horizontal cracks in the demineralization group [Figure 2]. The black dots, which were enamel porosities, were more clearly seen in the larger area on the demineralization group than the control group. In contrast, there were less porosities in the treatment group with fluoride varnish, CPP-ACPF varnish, and resin infiltration.

The porosity in both the control and demineralization groups had a honeycomb structure, but it was deeper in the demineralization group as compared with that in the control group as it had more contrast color. However, fluoride varnish application altered the porosity to look like layered-honeycomb, in which the porosity was covered by varnish material, and the varnish itself also forms a honeycomb coating layer. The enamel surface appeared to be covered by a layer of varnish in CPP-ACPF varnish treatment group, with no porosity found on some parts of the image. In resin infiltration treatment group, the porosity appeared to be defective, as characterized by the irregular porosity margin and the presence of interconnected porosity or damaged honeycomb. However, the size of each porosity appears to be smaller than that of the control group and seemed covered in some places.

\section{Shear strength test}

It was indicated that demineralization and resin infiltration groups had slightly higher mean of shear strength than control group, whereas treatment with fluoride varnish and CPP-ACPF varnish caused lower shear strength [Table 1]. However, no significant difference was found in shear strength among demineralization, control, and resin infiltration group, although the mean of demineralization group was higher than that in other groups [Table 2]. The shear strength in the treatment group of CPP-ACPF varnish was significantly lower than in the other four groups. Treatment with fluoride varnish also resulted in lower shear strength than in all groups but in CPP-ACPF varnish group.

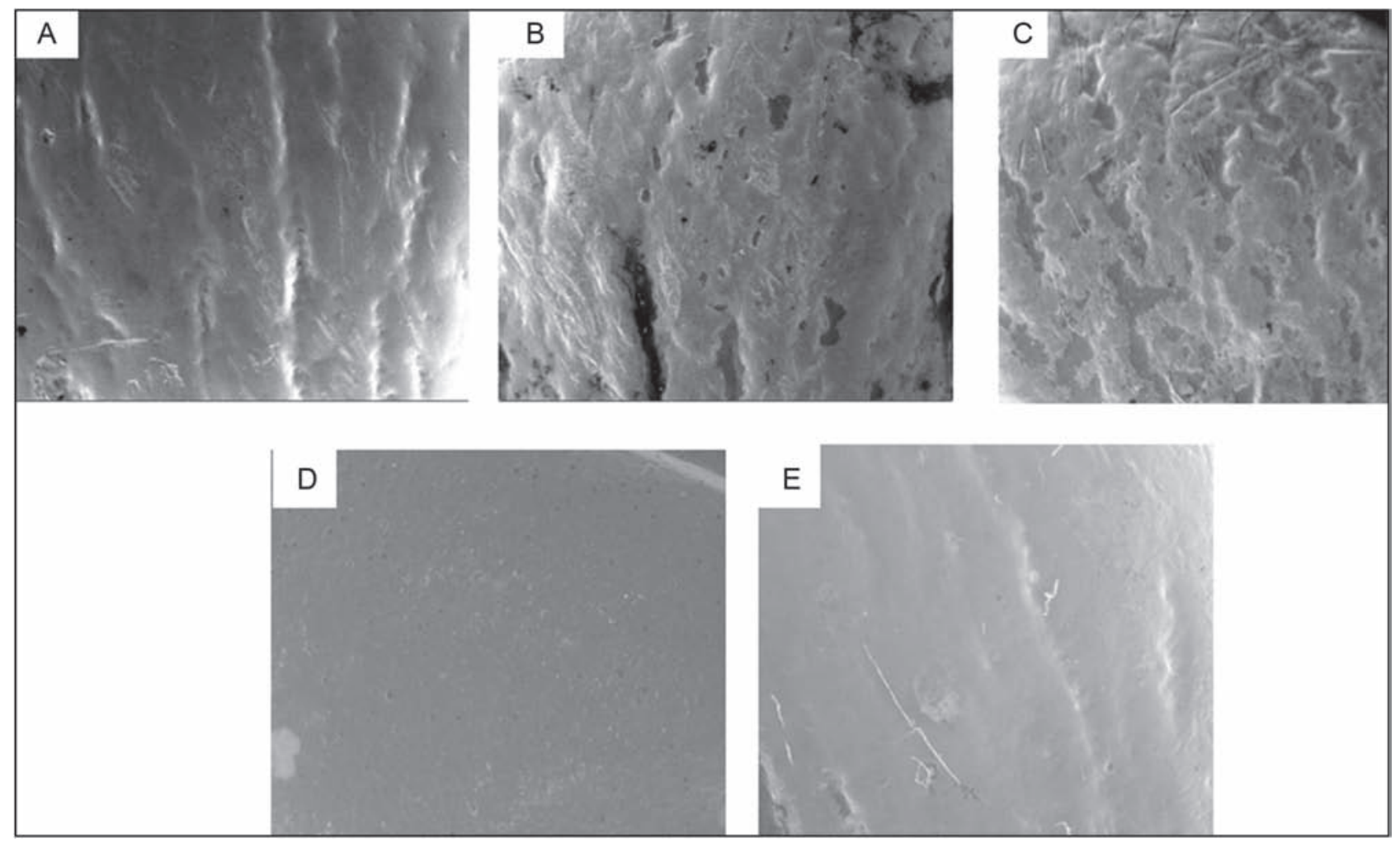

Figure 1: Enamel topography ( $\times 50$ SEM magnification) on group: (A) 1 (control), (B) 2 (demineralization), (C) 3 (fluoride varnish), (D) 4 (CPP-ACPF varnish), and (E) 5 (resin infiltration) 
Budipramana, et al: Different white-spot lesion treatment on orthodontics
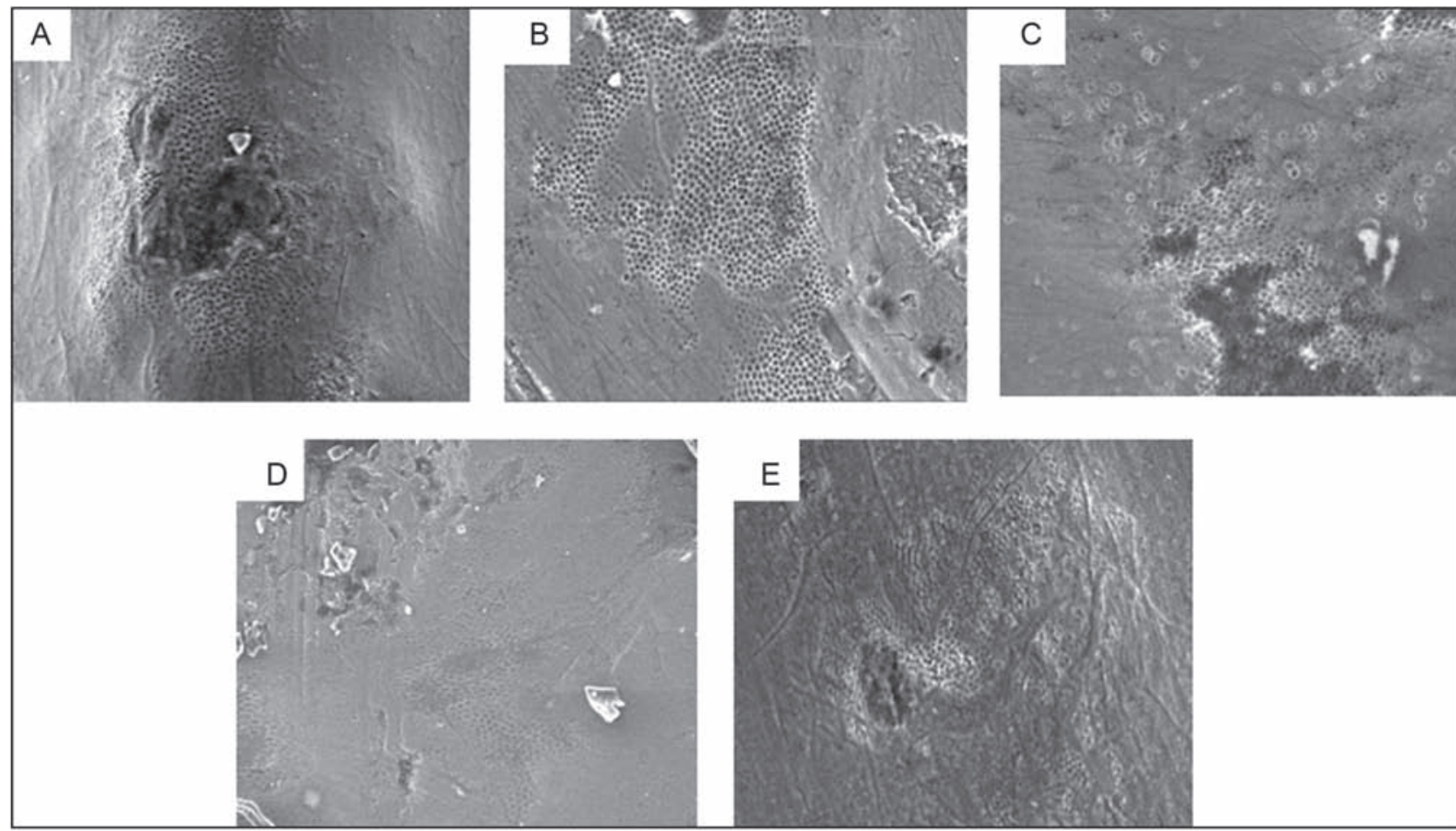

Figure 2: Enamel topography (×1000 SEM magnification) on group: (A) 1 (control), (B) 2 (demineralization), (C) 3 (fluoride varnish), (D) 4 (CPP-ACPF varnish), and (E) 5 (resin infiltration)

\begin{tabular}{|c|c|c|c|c|}
\hline \multirow[t]{2}{*}{ Group } & \multicolumn{2}{|c|}{ Shear force (kgf) } & \multicolumn{2}{|c|}{ Shear strength (MPa) } \\
\hline & Mean & SD & Mean & SD \\
\hline Control & 9.5833 & 1.1817 & 8.6960 & 1.0723 \\
\hline Demineralization & 10.4917 & 1.7468 & 9.5202 & 1.5850 \\
\hline Fluoride varnish & 6.3083 & 1.7489 & 5.7242 & 1.5869 \\
\hline CPP-ACPF varnish & 1.1378 & 0.5608 & 1.0324 & 0.5089 \\
\hline Resin infiltration & 10.283 & 1.0859 & 9.3312 & 0.9854 \\
\hline
\end{tabular}

Shear force was obtained by Shimatzu Autograph. Shear strength was shear force $(\mathrm{N})$ divided by bracket base area $\left(10.8 \mathrm{~mm}^{2}\right)$

Table 2: Significance score $(P)$ of shear strength by Tukey HSD statistical test

\begin{tabular}{lccccc}
\hline & Control & Demineralization & Fluoride varnish & CPP-ACPF varnish & Resin infiltration \\
\hline Control & - & 0.492 & $0.000^{*}$ & $0.000^{*}$ & 0.723 \\
Demineralization & & - & $0.000^{*}$ & $0.000^{*}$ & 0.996 \\
Fluoride varnish & & - & $0.000^{*}$ & $0.000^{*}$ \\
CPP-ACPF varnish & & & - & $0.000^{*}$ \\
Resin infiltration & & & & -
\end{tabular}

*Significant $(P<0.05)$

\section{Adhesive remnant index}

The scores of ARI obtained were ordinal data, so nonparametric statistics were used in the calculation [Table 3]. The ARI score mode in resin infiltration treatment group was the highest of all the other four groups.

On the basis of the Kruskal-Wallis test followed by Mann-Whitney $U$ test [Table 4], it can be concluded

\section{Table 3: ARI evaluation score mode}

\begin{tabular}{lc}
\hline Group & Mode \\
\hline Control & 1 \\
Demineralization & 1 \\
Fluoride varnish & 1 \\
CPP-ACPF varnish & 1 \\
Resin infiltration & 2 \\
\hline
\end{tabular}


Budipramana, et al.: Different white-spot lesion treatment on orthodontics

\begin{tabular}{|c|c|c|c|c|c|}
\hline & Control & Demineralization & Fluoride varnish & CPP-ACPF varnish & Resin infiltration \\
\hline Control & - & 0.397 & 0.284 & 0.114 & $0.014^{*}$ \\
\hline Demineralization & & - & 0.065 & $0.030^{*}$ & 0.089 \\
\hline Fluoride varnish & & & - & 0.386 & $0.000^{*}$ \\
\hline CPP-ACPF varnish & & & & - & $0.000^{*}$ \\
\hline
\end{tabular}

*Significant $(P<0.05)$

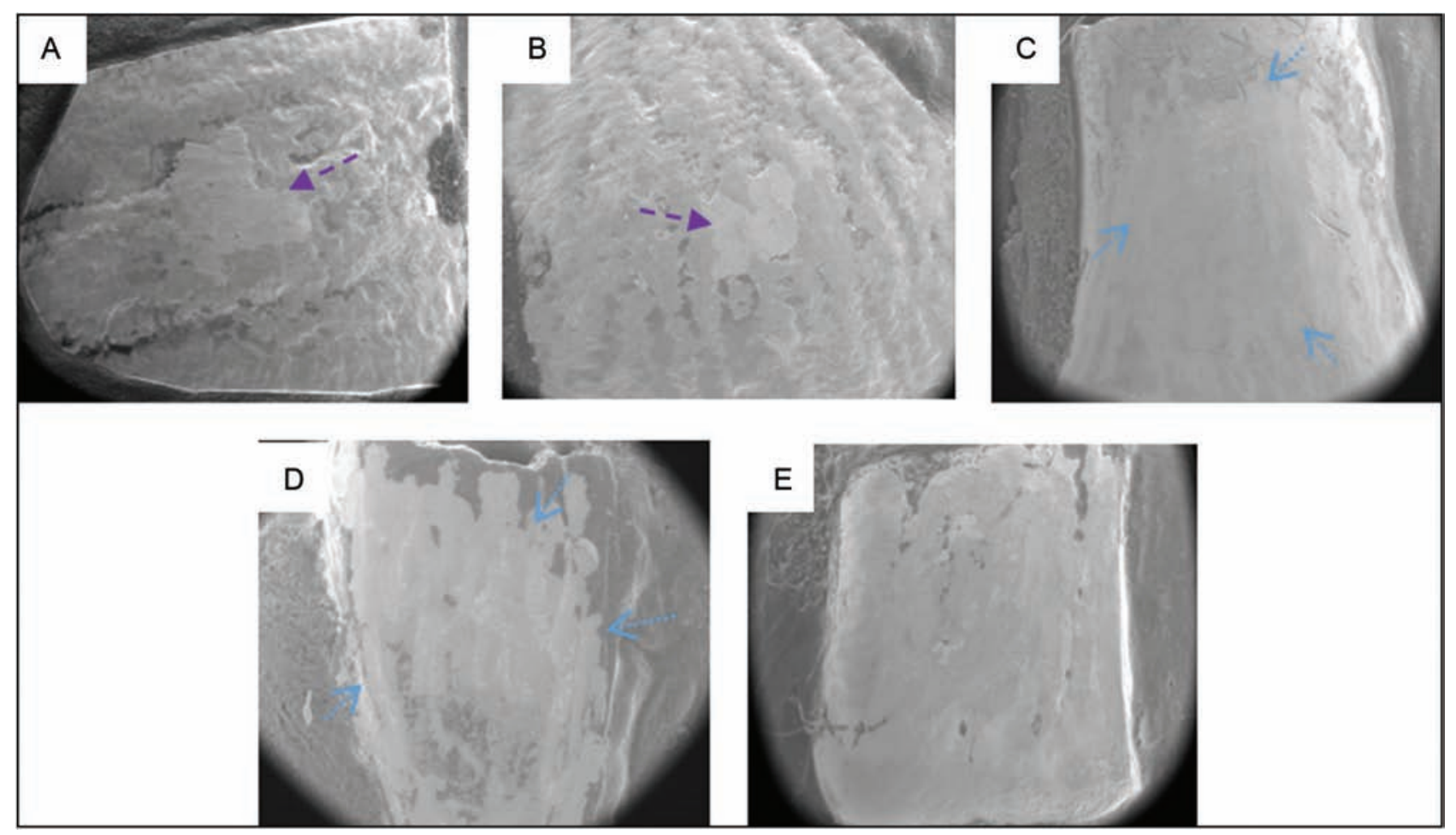

Figure 3: Adhesive remnant and the missing of varnish layer after debonding and polishing ( $\times 30$ SEM magnification) on group: (A) 1 (control), (B) 2 (demineralization), (C) 3 (fluoride varnish), (D) 4 (CPP-ACPF varnish), and (E) 5 (resin infiltration). Note: the purple arrow shows adhesive remnant and the blue arrow shows the disappearance of varnish layer

that the ARI score mode in resin infiltration treatment group was the highest of all the other four groups. In addition, the ARI score was found to be significantly higher in demineralization group than that of CPP-ACPF varnish group.

\section{Effects on enamel surface after debonding and polishing}

After debonding and polishing process, an observation was made using SEM to see enamel surface alteration further. Figure 3 shows that there was some adhesive material left in control and demineralization group, even after visually checked polishing had been performed. In contrast, there was no visible residual adhesive in the other three treatment groups. However, a layer of varnish material disappeared. Treatment with resin infiltration enabled the enamel surface to appear as smooth as before bracket placement.

On higher magnification, it was revealed that there was residual adhesive on the enamel surface of all samples
[Figure 4]. Similar to the observations before bracket placement, vertical depressions were clearly visible, with deeper depressions in the demineralization group. In contrast, enamel damage was found on resin infiltration treatment group, as marked by the presence of black areas on several spots.

A greater amount of adhesive remnant was found in the demineralization than in control group, whereas that in resin infiltration group was higher than in the demineralization group [Figure 5]. They appeared only as white dots in fluoride varnish treatment group. No residual adhesive was seen in CPP-ACPF varnish treatment group.

\section{Discussion}

In this study, the SEM analysis revealed that demineralization process resulted in more enamel cracks than in the control group. In addition, enamel surface got rougher, whitish, with deeper depression than that of 


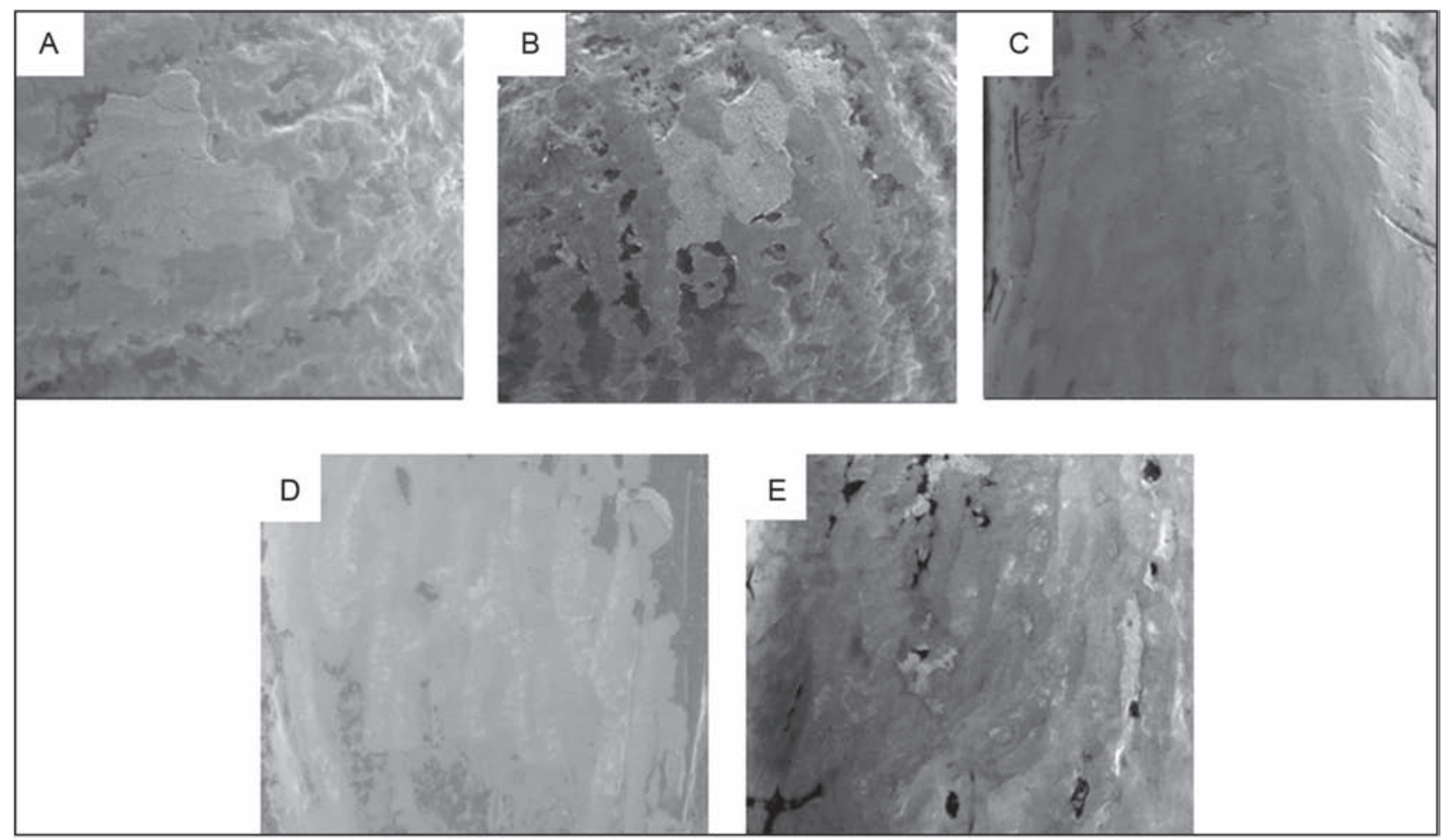

Figure 4: Enamel topography after debonding dan polishing ( $\times 50$ SEM magnification) on group: (A) 1 (control), (B) 2 (demineralization), (C) 3 (fluoride varnish), (D) 4 (CPP-ACPF varnish), and (E) 5 (resin infiltration)

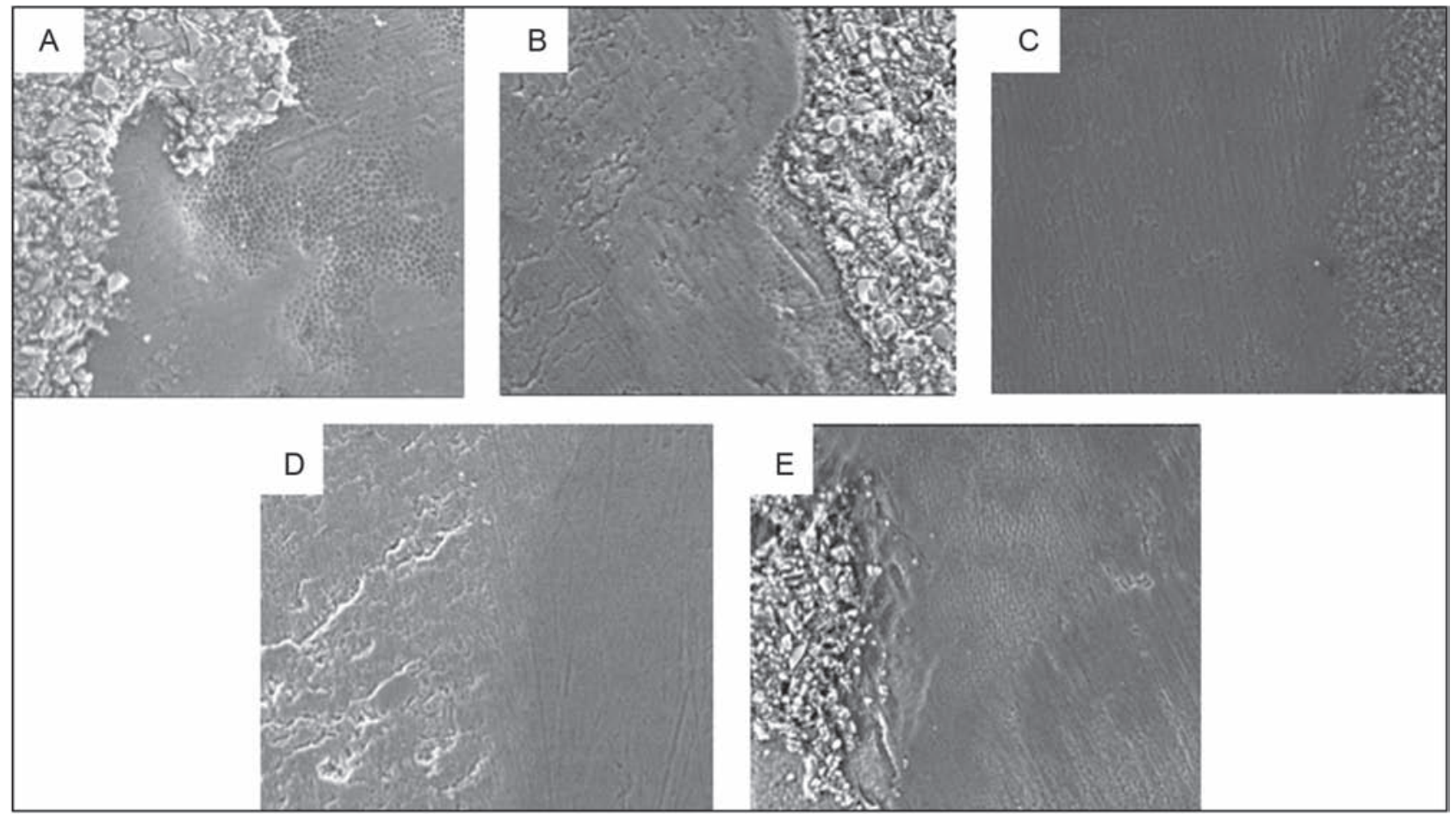

Figure 5: Enamel topography ( $\times 1000$ SEM magnification) after debonding and polishing on group: (A) 1 (control), (B) 2 (demineralization), (C) 3 (fluoride varnish), (D) 4 (CPP-ACPF varnish), and (E) 5 (resin infiltration)

control group. Enamel porosities were deeper as observed with greater magnification. This result was consistent with the study of human premolars by Nhu et al., ${ }^{[12]}$ Honeycomb appearance, which was formed by deep enamel porosities, seen after the immersion in demineralization solution confirmed the occurence of enamel's mineral. Other 
studies by Worawongvasu ${ }^{[13]}$ and Hicks et al. ${ }^{[14]}$ also stated that demineralization process led to partial disappearance of central prisms nucleus on the enamel surface, whereas the peripheral prisms remained, which seen as honeycomb appearance.

Smoother enamel topography was obtained after applying white-spot treatment materials. Varnish material could fill the depression caused by demineralization process. CPP-ACPF varnish layered enamel surface with a homogenous substance, and thus it looked smooth. This result was consistent with experiments by Poggio et al. ${ }^{[15]}$ and Kucuk et al. ${ }^{[16]}$ using CPP-ACP in the form of paste. SEM analysis revealed that the porosities were perfectly sealed in some parts of the tooth by CPP-ACP material. Application of resin infiltration made enamel surface looked smoother than normal tooth. ${ }^{[17]}$ The appearance of destructed pores might be because of etching process in resin infiltration, but some pores seemed to be sealed by resin material which filled the pores.

There was no significant difference in shear strength in control and demineralized samples. This was inconsistent with the studies by Baka et al. ${ }^{[4]}$ and Baysal and Uysal, ${ }^{[18]}$ who stated that early caries (demineralized) tooth had lower shear strength than normal tooth. The difference could be caused by different bonding agent used. In those studies, composite resin was used, whereas in this study RMGIC was used. In addition, exposed porosity resulting from demineralization process was also required for bonding agent's resin tag penetration. ${ }^{[19]}$ Treatment with resin infiltration produced greater shear strength than that in demineralization group, although not significant. Penetration of resin tag into enamel pores followed by chemical bonding of RMGIC bonding agent with resin infiltration made brackets adhere stronger to enamel. On the contrary, the shear strength of varnish group was found to be significantly lower than that of control group. It may be caused by the direct bracket placement to varnish layer without being polished with pumice, so that it did not bond with remineralized enamel surface.

This result was consistent with the experiments by Bayrak et al. ${ }^{[20]}$ and Endo et al., ${ }^{[2]]}$ in which fluoride varnish was applied before bracket attachment to human tooth samples using RMGIC bonding agent. Nonetheless, this result was inconsistent with a study by Nhan et al., ${ }^{[22]}$ in which fluoride varnish application did not contribute to the difference of shear strength of brackets bonded with composite resin bonding agent and phosphoric acid etch. However, a study by Viana et al. ${ }^{[23]}$ concluded that low fluoride level of enamel did not significantly decrease the bond strength, for example, the use of fluoride-containing pumice. On the contrary, the use of fluoride-containing self-etch decreased the bond strength. It could be caused by the decrease of resin tag penetration depth.
Nonetheless, in an experiment using acidulated phosphate fluoride (APF)-containing etch, there was no change in the bonding strength. ${ }^{[2]}$

In this study, application of CPP-ACPF before bonding process resulted in low shear strength. It was contradictory to a study by Cehreli et al. ${ }^{[25]}$ who stated that application of CPP-ACPF paste to human teeth before bonding process with composite resin and etch-and-rinse system and self-etch only insignificantly decreased the shear strength. Moreover, Karabekiroglu et al. ${ }^{[26]}$ found that the application of CPP-ACPF before or after etching process did not affect the shear strength. This difference might be because the CPP-ACPF preparation used in that study was in paste form, while in this study varnish form was used. In addition, the bonding agent used in those studies was composite resin. In this experiment, the average shear strength of control, of demineralization, and of resin infiltration treatment group was in the suggested range as $6-8 \mathrm{MPa} .{ }^{[27,28]}$ The shear strength after varnish treatment was lower than the suggested strength; thus, the varnish application should be avoided before orthodontic treatment.

Lower ARI scores allowed orthodontists to clean residual adhesive material faster with minimum enamel destruction. ${ }^{[29]}$ In this study, ARI scores were only observed on enamel surface to determine post-debonding enamel quality. Basically, ARI scores in RMGIC bonding agent revealed that the bonding failure occurred on the interface of enamel surface-bonding agent, whereas the bonding failure of composite resin bonding agent was on the interface of adhesive and brackets. ${ }^{[27]}$

In this experiment, the ARI scores were found to be significantly higher in resin infiltration treatment group. It supported a theory that the chemical bond between resin infiltration and resin in bonding agent occurred, thus enabling resin tag to penetrate deeper into the porosity after hydrochloric acid etching process while applying resin infiltration. In the other four groups, no significant difference was found between ARI scores, probably because of manual score observation (with eyes). The ARI observation method performed with different magnifications had different reliability results. In greater magnification (more than 20 times), lower ARI scores tended to decrease and higher scores increase as compared with manual observation. ${ }^{[25]}$ Nonetheless, in reality, orthodontists manually observed residual adhesive material after debonding.

After polishing, the enamel surface was reobserved by SEM to determine any enamel destruction. In greater magnification of SEM observation, there was still residual adhesive material left in all samples. Consistent with the increase of shear strength mean, although statistically insignificant, the residual adhesive material after resin 
infiltration application was found to be thicker than that of the other groups. However, enamel destruction also occurs worse in resin infiltration treatment group. New horizontal cracks were found in demineralization group. On the contrary, in the varnish treatment group, there was little residual adhesive material, and varnish layer disappeared as wide as the brackets, exposing relatively smooth enamel contour underneath. It might be caused by the bracket attachment on varnish layer, not directly to remineralized enamel surface, which resulted in low shear strength and ARI scores.

On the basis of the result of this study, the shear strength of RMGIC bonding agent on demineralized enamel was not significantly different from that in normal enamel. It indicated that RMGIC could be used as an orthodontic bonding agent on demineralized enamel without reducing the bond strength. Therefore, treatment on WSL before short-term fixed orthodontic treatment might not be required. In addition, RMGIC could also reduce the risk of advancing demineralization lesion because of its fluoride content.

The use of varnish could repair enamel surface destruction caused by demineralization process, but it caused the shear strength to be very low as the brackets bonded with varnish layer. Further studies using XPS (X-ray photoelectron spectroscopy) were needed to determine the extent of remineralization in underlying enamel. XPS observation had some benefits: pretreatment on samples was not needed (unlike SEM analysis) so that it would not cause further alteration to enamel surface and atomic content in the form of chemical elements can be measured. ${ }^{[23,30]}$ However, if the underlying enamel had been remineralized, polishing using pumice was suggested to be performed before bracket placement to remove residual varnish layer, and thus the bonding material could attach directly to the enamel surface. The varnish application could also be performed for remineralizing tooth surface after debonding because of demineralization and enamel damage occurred during orthodontic treatment and polishing process. ${ }^{[31,32]}$

The limitation of this study was that no measurement was carried out on remineralization depth in bovine enamel samples, layer thickness of applied fluoride varnish, CPP-ACPF varnish, and resin infiltration, and the depth of enamel lost during polishing. These made the real degree of enamel destruction could not be measured in a three-dimensional way.

\section{ConcLusion}

Pretreatment with resin infiltration is recommended to be performed before bracket bonding on WSL; however, it may need to be reapplied after debonding and polishing to conceal enamel morphology damage formed after polishing. In addition, RMGIC bonding agent can also be used on WSL without any pretreatment.

\section{Ethical policy and institutional approval consent}

This study was ethically approved by Faculty of Dentistry Airlangga University Dental Hospital (Protocol number HRECC.FODM/XII/2018).

\section{Data availability statement}

The data set used in this study is available on request on corresponding author mail.

\section{Acknowledgement}

The authors would like to thank Faculty of Dental Medicine and Dental Hospital of Universitas Airlangga, Surabaya, East Java, Indonesia.

\section{Financial support and sponsorship}

Nil.

\section{Conflicts of interest}

There are no conflicts of interest.

\section{References}

1. Hedayati Z, Farjood A. Evaluation of microleakage under orthodontic brackets bonded with nanocomposites. Contemp Clin Dent 2018;9:361-6.

2. Sundararaj D, Venkatachalapathy S, Tandon A, Pereira A. Critical evaluation of incidence and prevalence of white spot lesions during fixed orthodontic appliance treatment: A meta-analysis. J Int Soc Prev Community Dent 2015;5:433-9.

3. Sandra CD, Maria D, Ingrid MD, Vanessa HD, Katia VR. Preventing and arresting the appearance of white spot lesions around the bracket by applying fluoride varnish: A systematic review. Dentistry 2018;8:500.

4. Baka ZM, Akin M, Ileri Z, Basciftci FA. Effects of remineralization procedures on shear bond strengths of brackets bonded to demineralized enamel surfaces with self-etch systems. Angle Orthod 2016;86:661-7.

5. Uysal T, Baysal A, Uysal B, Aydınbelge M, Al-Qunaian T. Do fluoride and casein phosphopeptide-amorphous calcium phosphate affect shear bond strength of orthodontic brackets bonded to a demineralized enamel surface? Angle Orthod 2011;83:490-5.

6. Restrepo M, Bussaneli DG, Jeremias F, Cordeiro RC, Magalhães AC, Palomari Spolidorio DM, et al. Control of white spot lesion adjacent to orthodontic bracket with use of fluoride varnish or chlorhexidine gel. Sci World J 2015;2015:218452.

7. Perrini F, Lombardo L, Arreghini A, Medori S, Siciliani G. Caries prevention during orthodontic treatment: In-vivo assessment of high-fluoride varnish to prevent white spot lesions. Am J Orthod Dentofacial Orthop 2016;149:238-43.

8. Mayne RJ, Cochrane NJ, Cai F, Woods MG, Reynolds EC. In vitro study of the effect of casein phosphopeptide amorphous calcium fluoride phosphate on iatrogenic damage to enamel during orthodontic adhesive removal. Am J Orthod Dentofacial Orthop 2011;139:e543-51.

9. Naidu E, Stawarczyk B, Tawakoli PN, Attin R, Attin T, Wiegand A. Shear bond strength of orthodontic resins after caries infiltrant preconditioning. Angle Orthod 2013;83:306-12.

10. Ten Cate JM, Duijsters PP. Alternating demineralization and remineralization of artificial enamel lesions. Caries Res 1982;16:201-10. 
11. Artun J, Bergland S. Clinical trials with crystal growth conditioning as an alternative to acid-etch enamel pretreatment. Am J Orthod 1984;85:333-40.

12. Nhu NVT, Hong TPT, Quynh AL, Minh ST, Thu PNT. The effect of casein phosphopeptide-amorphous calcium fluoride phosphate on the remineralization of artificial caries lesions: An in vitro study. J Dent Indones 2017:24:45-49.

13. Worawongvasu R. A scanning electron microscopic study of enamel surfaces of incipient caries. Ultrastruct Pathol 2015;39:408-12.

14. Hicks J, Wu J, Flaitz CM. Effect of xylitol and fluoride dentifrices on human enamel surfaces following acid-etching: Scanning electron microscopic study. Pediatr Dent Care 2016;1:127-33.

15. Poggio C, Lombardini M, Vigorelli P, Ceci M. Analysis of dentin/ enamel remineralization by a CPP-ACP paste: AFM and SEM study. Scanning 2013;9999:1-9.

16. Kucuk EB, Malkoc S, Demir A. Microcomputed tomography evaluation of white spot lesion remineralization with various procedures. Am J Orthod Dentofacial Orthop 2016;150:483-90.

17. Arnold WH, Meyer AK, Naumova EA. Surface roughness of initial enamel caries lesions in human teeth after resin infiltration. Open Dent J 2016;10:505-15

18. Baysal A, Uysal T. Do enamel microabrasion and casein phosphopeptide-amorphous calcium phosphate affect shear bond strength of orthodontic brackets bonded to a demineralized enamel surface? Angle Orthod 2012;82:36-41

19. Papargeorgiou SN, Pandis N. Clinical evidence on orthodontic bond failure and associated factors. In: Eliades T, Brantley W, editors. Orthodontic Applications of Biomaterials: A Clinical Guide. 1st ed. Duxford, UK: Elsevier; 2017. p. 191,197-8.

20. Bayrak S, Tuloglu N, Ozer F, Blatz MB. Effect of fluoride varnish with added casein phophopeptide-amorphous calcium phosphate on bond strength to enamel. J Adhes Sci Technol 2016;31:581-90.

21. Endo T, Ishida R, Komatsuzaki A, Sanpei S, Tanaka S, Sekimoto T. Effects of long-term repeated topical fluoride applications and adhesion promoter on shear bond strengths of orthodontic brackets. Eur J Dent 2014;8:431-6.

22. Nhan RT, Xu X, Yu Q, Ballard R, Armbruster P. The effect of topical fluoride varnish on the shear bond strength of orthodontic brackets. Aust Orthod J 2015;31:14-9.
23. Viana PS, Orlandi MO, Pavarina AC, Machado AL, Vergani CE. Chemical composition and morphology study of bovine enamel submitted to different sterilization methods. Clin Oral Investig 2018;22:733-44

24. Cai Z, Iijima M, Eliades T, Brantley W. Frequent handling mistakes during bonding. In: Eliades T, Brantley W, editors. Orthodontic Applications of Biomaterials: A Clinical Guide. 1st ed. Duxford, UK: Elsevier; 2017. p. 171-2.

25. Cehreli SB, Sar C, Polat-Özsoy O, Unver B, Ozsoy S. Effects of a fluoride-containing casein phosphopeptide-amorphous calcium phosphate complex on the shear bond strength of orthodontic brackets. Eur J Orthod 2012;34:193-7.

26. Karabekiroglu S, Akdemir I, Kahraman FB. Influence of CPPACPF paste and fluoride varnish on bracket shear bond strength of two adhesive systems. Int J Appl Dent Sci 2017;3:228-32.

27. Scribante A, Contreras-Bulnes R, Montasser MA, Vallittu PK. Orthodontics: bracket materials, adhesives systems, and their bond strength. Biomed Res Int 2016;2016:1329814.

28. Eliades T, Brantley W. Bond strength and its limited role in assessing efficacy. In: Eliades T, Brantley W, editors. Orthodontic Applications of Biomaterials: A Clinical Guide. 1st ed. Duxford, UK: Elsevier; 2017. p. 179-87.

29. Sessa T, Civović J, Pajević T, Juloski J, Beloica M, Pavlović V, et al. Scanning electron microscopic examination of enamel surface after fixed orthodontic treatment: in-vivo study. Srp Arh Celok Lek 2012;140:22-8.

30. Santak V, Vesel A, Zaplotnik R, Biscan M, Milosevic S. Surface treatment of human hard dental tissues with atmospheric pressure plasma jet. Plasma Chem Plasma Process 2017;37: 401-13.

31. He T, Li X, Dong Y, Zhang N, Zhong Y, Yin W, et al. Comparative assessment of fluoride varnish and fluoride film for remineralization of postorthodontic white spot lesions in adolescents and adults over a 6-month period: A single-center, randomized controlled clinical trial. Am J Orthod Dentofacial Orthop 2016;149:810-9.

32. Patcas R, Eliades T. Enamel alterations due to orthodontic treatment. In: Eliades T, Brantley W, editors. Orthodontic Applications of Biomaterials: A Clinical Guide. 1st ed. Duxford, UK: Elsevier; 2017. p. 224-33. 